\title{
KINEMATICS OF THE ROLLING SPHERE AND QUANTUM SPIN*
}

\author{
ANTHONY M. BLOCH ${ }^{\dagger}$ AND ALBERTO G. ROJO $\ddagger$
}

\begin{abstract}
We consider the kinematics and control of a sphere rolling of a curved surface and analyze its rotation by mapping the system to the precession of a spin $1 / 2$ in a magnetic field of variable magnitude and direction. This mapping is useful in understanding the role of the geometrical phase and generalizes the kinematic control problem of a ball rolling on a plane.
\end{abstract}

1. Introduction. In this paper we consider the geometry and kinematics of a ball rolling on a curved surface and its relationship to kinematic control. In particular we consider a question similar to that posed in the title of reference [8]: How much does a sphere rotate when rolling on a curved surface? In [8], the old problem of the rotation of a torque free, non-spherical body is reanalyzed. The angle of rotation is identified to have two components, one dynamical and one geometrical (the so called Berry phase), independent of the time elapsed during the rotation. This analysis can also be generlalized to understand the problem of controlling the orientation of a satellite with momentum wheels (see [3]).

Here we consider a related but different problem: a sphere is made to roll without slipping on a given curve $\Gamma$ on a surface. The question is, if the sphere completes a circuit, what is the rotation matrix connecting the initial and final configuration of the sphere? The problem we are considering is therefore a kinematic rather than a dynamic one: the trajectory of the contact point of the sphere and the surface is dictated externally and the rolling constraint is imposed. We make contact with recent approaches that consider the same problem [9, 10] (but on a plane), and in particular, we address the nice question posed by Brockett and Dai [11]: a sphere lies on a table and is made to rotate by a flat plane on top of it, parallel to the table. The question is: if every point of the plane describes a circle, what is the trajectory and motion of the sphere? Moreover we show how to generalize this analysis to the more general problem of a ball rolling on a curved surface. We show in this general setting that rolling causes the geometrical phase to vanish when one completes a closed curve and we calculute the value of the remaining geometric phase.

The problem is a particular (but important) case of the control of underactuated kinematic control systems of the form $\dot{x}=A(x) u$ where $x \in R^{n}, u \in R^{m}, m<n$ and $A$ is $n \times n$. The prototypical example of this problem is the nonholonomic integrator or Heisbenberg system as analyzed in [7] (see also [17] and e.g. [6]). A related key

\footnotetext{
*Dedicated to John Baillieul on the Occasion of His 65th Birthday.

$\dagger$ Department of Mathematics, University of Michigan, Ann Arbor, MI 48109. E-email: abloch@umich.edu

‡Department of Physics, Oakland University, Rochester, MI 48309. E-mail: rojo@oakland.edu
} 
example is the kinematic control of rigid body systems with two controls. Some of the most interesting early work in this area was discussed in the work of Baillieul (see [1] and [2]). There is of course much other literature in this area, see e.g. the references in [17]. In the current setting we analyze a uniform sphere (perfectly symmetric rigid body) constrained to move a surface. One can think here too of the motion as being generated by moving a plane on top of the sphere.

A novel feature here is that we treat the problem by exploiting its isomorphism to the precession of a spin $1 / 2$ in a time-dependent magnetic field. In the mapping, the arc length of the curve plays the role of time. For rolling on a plane the magnitude of the magnetic field is $1 / R$ with $R$ the radius of the sphere, and the direction of the magnetic field is that of the instantaneous angular velocity of the rolling sphere. For a curved surface the normal curvature and the torsion of the curve affect the value of the effective magnetic field. Closely related to the present paper is the use of the isomorphism between classical dynamics and that of a spin $1 / 2$ by Berry and Robbins in Ref. [12], especially their classical view of the Landau-Zener [14] problem. In a related paper, see [5], we use the rolling analogy to analyze the Lanau-Zener problem. We discuss this idea briefly at the end of this paper and we hope to extend this idea further to the control of spins.

The precession of a spin $1 / 2$ is widely treated in the literature and one can borrow those results to acquire an intuition for the rolling sphere. Conversely, since a rolling sphere is a tangible physical problem, the present treatment is also useful in understanding spin precession, Berry's phases and it's classical counterpart, Hannay's angle [13].

2. Rolling on a Plane and Quantum Precession. Consider a sphere of radius $R$ rolling on a curve $\Gamma$ on a plane. We define a local triad of unit vectors at the contact point (the so called Darboux frame [15]): the tangent $\mathbf{t}$ to $\Gamma$, the normal $\mathbf{n}$ to the surface, and $\mathbf{u}=\mathbf{n} \times \mathbf{t}$, the tangent normal. For rolling on a plane $\mathbf{n}$ is a constant vector, and the velocity of the center of the sphere is along the tangent to the curve. This situation will change for rolling on a curved surface, but, as we will see, the general idea of the mapping to a precessing spin is the same.

The translational velocity of the sphere is $\mathbf{V}=\mathbf{t} V(t)$ and the rolling constraint means that the instantaneous velocity at the contact point is zero [16]:

$$
\vec{\omega} \times(\mathbf{n} R)=\mathbf{V}=\mathbf{t} V(t)
$$

with $\vec{\omega}$ the angular velocity and $R$ the radius of the sphere. This equation is nonintegrable and constitutes a paradigmatic nonholonomic constraint [17].

Taking the cross product with $\mathbf{n}$ on both sides of the above equation we have

$$
\vec{\omega}=\frac{V(t)}{R} \mathbf{n} \times \mathbf{t} \equiv \frac{V(t)}{R} \mathbf{u} .
$$


Notice that in the above equation we have used the "no twist" condition $\vec{\omega} \cdot \mathbf{n}=0$, that is, we are consider rolling without an instantaneous rotation along the normal.

The instantaneous velocity $\dot{\mathbf{X}}$ of a point of coordinate $\mathbf{X}$ (with respect to the center of the sphere) on the surface of the sphere is

$$
\dot{\mathbf{X}}=\vec{\omega} \times \mathbf{X}=\frac{V(t)}{R} \mathbf{u} \times \mathbf{X}
$$

Now we rewrite $V(t)=d s / d t$ where $s$ is the arc length of the curve $\Gamma(t)$, and (3) becomes

$$
\frac{d \mathbf{X}}{d s}=\frac{\mathbf{u}}{R} \times \mathbf{X}
$$

Here we may regard $\mathbf{u}$ as a control vector and the equation (4) as representing a fully actuated kinematic control problem in three-space.

If we regard $\mathbf{X}=(x, y, z)$ as a magnetic moment, the above equation describes its precession in the presence of a magnetic field $\mathbf{B}=-\frac{1}{R}\left(u_{x}, u_{y}, u_{z}\right)=-\vec{\omega}_{s}$ of constant magnitude $1 / R$. (We use here the notation $\vec{\omega}_{s}$ which has units of inverse arc length to distinguish it from $\vec{\omega}$ which has units of inverse time.) The direction of $\mathbf{B}$ is $-\mathbf{u}$, and varies with $s$, the arc length, which plays the role of time. If the rolling is on a horizontal plane, then $B_{z}=0$, but we keep this notation to make contact with the rolling on an arbitrary surface.

There is an isomorphism between the rolling sphere written in this way with a spin $1 / 2$ precessing in this magnetic field. This isomorphism can be seen clearly if, (using $\mathbf{B}=-\vec{\omega}$ ) we rewrite Equation (4) in the form

$$
\frac{d}{d s}\left(\begin{array}{c}
x \\
y \\
z
\end{array}\right)=\left(\begin{array}{ccc}
0 & B_{z} & -B_{y} \\
-B_{z} & 0 & B_{x} \\
B_{y} & -B_{x} & 0
\end{array}\right)\left(\begin{array}{l}
x \\
y \\
z
\end{array}\right)
$$

which is the same as the following equation of motion for two complex numbers $a$ and $b$ (we write $s$ instead of $t$ for time in order to keep the analogy)

$$
i \frac{d}{d s}\left(\begin{array}{c}
a \\
b
\end{array}\right)=-\frac{1}{2}\left(\begin{array}{cc}
B_{z} & B_{x}-i B_{y} \\
B_{x}+i B_{y} & -B_{z}
\end{array}\right)\left(\begin{array}{l}
a \\
b
\end{array}\right),
$$

with the identification

$$
\begin{aligned}
& x \equiv a b^{*}+b a^{*} \\
& y \equiv i\left(a b^{*}-b a^{*}\right) \\
& z \equiv a a^{*}-b b^{*} .
\end{aligned}
$$

Again note that from the control point of view we may regard $\mathbf{B}=-\frac{1}{R}\left(u_{x}, u_{y}, u_{z}\right)$ $=-\vec{\omega}$ as the control vector of a fully actuated kinematic system. 
The real numbers $(x, y, z)$ represent the coordinates of a point on the surface of the sphere referred to a coordinate system fixed in space (that is, not rotating), and whose origin is in the center of the sphere. The above mapping is certainly possible because of the $s u(2)-s o(3)$ isomorphism [18].

Equation (6) is Schrödinger's equation for the spinor $\chi=(a, b)$ in the presence of a magnetic field $\mathbf{B}$ :

$$
i \frac{d}{d s} \chi=-\mathbf{B} \cdot \mathbf{S} \chi \equiv H \chi
$$

where $\hbar=1$ and $H$ is the Hamiltonian. Also, the vector $\mathbf{S}=\frac{1}{2}\left(\sigma_{x}, \sigma_{y}, \sigma_{z}\right)$ is the spin operator, and $\sigma_{i}$ are Pauli's matrices. Notice that in this mapping, the magnetic fields and the frequencies have units of inverse length,

Equation (7) implies that we can extract the behavior of the rolling sphere as a function of arc length by solving the motion of a spin $1 / 2$ in a time-varying magnetic field. To our knowledge the equivalence between the motion of rigid body and a twolevel system (a spin 1/2), in the form of the mapping of Eq. (7) was first pointed out by Feynman, Vernon and Hellwarth [19] and later discussed several times [22]. Earlier, Bloch [20] had derived the precession equation for the density matrix of spin $1 / 2$ and therefore the points $(x, y, z)$ that result from the mapping from spinors are called the Bloch sphere.

One of the novelties of the present paper is to discuss the rolling using the arc length as time and identifying the isomorphism between the rolling sphere and the quantum spin in exactly solvable cases.

It is also important to note that, using the isomorphism between $\mathbb{R}^{3}$ and $s o(3)$ given by

$$
\left(\begin{array}{c}
\hat{x} \\
y \\
z
\end{array}\right)=\left(\begin{array}{ccc}
0 & z & -y \\
-z & 0 & x \\
y & -x & 0
\end{array}\right)
$$

equation (4) may be written as

$$
\frac{d \hat{\mathbf{X}}}{d s}=\left[\frac{\hat{\mathbf{u}}}{R}, \hat{\mathbf{X}}\right] .
$$

This is a kinematic control problem on an adjoint orbit of the orthogonal group $S O(3)$ - in this case the sphere.

One can pose the following optimal control problem for this system:

$$
\min _{u(\cdot)} \int_{0}^{S}\left(u_{x}^{2}+u_{y}^{2}+u_{z}^{2}\right) d s,
$$

subject to the equation (10) and the endpoint conditions: $\hat{\mathbf{X}}(0)=\hat{\mathbf{X}}_{0}$ and $\hat{\mathbf{X}}(S)=\hat{\mathbf{X}}_{S}$, This optimal control can be solved in a number of ways, yielding geodesics on the 
sphere. In particular one can show that one obtains constant controls (or magnetic fields in this case.) leading to simple conjugation on the sphere.

One can in fact show that the full solution to the problem is of the coupled double bracket form (see [4])

$$
\begin{aligned}
\frac{d \hat{\mathbf{X}}}{d s} & =[\hat{\mathbf{X}},[\hat{\mathbf{P}}, \hat{\mathbf{X}}]] \\
\frac{d \hat{\mathbf{P}}}{d s} & =[\hat{\mathbf{P}},[\hat{\mathbf{P}}, \hat{\mathbf{X}}]]
\end{aligned}
$$

where $\hat{\mathbf{u}}=[\hat{\mathbf{P}}, \hat{\mathbf{X}}]$ and $\hat{\mathbf{P}}$ is matrix Lagrange multiplier. One can check that the control vector is constant along the flow.

3. Constant Magnetic Field/Constant Control. Consider the simplest case of constant magnetic field or constant control. We choose $\mathbf{B}=B_{0} \hat{\mathbf{k}}$, constant in the $+z$ direction. This corresponds to the sphere rolling on a vertical plane. Eq (6) becomes:

$$
i \frac{d}{d s}\left(\begin{array}{c}
a \\
b
\end{array}\right)=-\frac{1}{2}\left(\begin{array}{cc}
B_{0} & 0 \\
0 & -B_{0}
\end{array}\right)\left(\begin{array}{l}
a \\
b
\end{array}\right),
$$

with solutions:

$$
\left(\begin{array}{c}
a(s) \\
b(s)
\end{array}\right)=\left(\begin{array}{c}
e^{i s B_{0} / 2} a(0) \\
e^{-i s B_{0} / 2} b(0)
\end{array}\right) .
$$

Substituting (14) in (7) we obtain:

$$
\begin{aligned}
& x(s)=x(0) \cos \left(B_{0} s\right)+y(0) \sin \left(B_{0} s\right) \\
& y(s)=y(0) \cos \left(B_{0} s\right)-x(0) \sin \left(B_{0} s\right) \\
& z(s)=z(0),
\end{aligned}
$$

which means that the sphere is rotating clockwise around a constant axis in the $z$ direction. This corresponds to $\vec{\omega}$ in the $-z$ direction. In other words, a constant magnetic field in the $z$ direction corresponds to the sphere moving in a straight line in the $x y$ plane, rolling on a vertical wall. The same situation applies if a constant field is directed in any other orientation.

Thus from the previous section we have

THEOREM 1. Rolling of the sphere on a straight line on any plane corresponds to geodesic motion in $s$.

4. The Planar Field and Rotation in a Circle. Consider a magnetic field varying on the $x y$ plane as $\mathbf{B}=B(\cos \alpha s, \sin \alpha s, 0)$. This corresponds to $\mathbf{u}$ rotating with the same frequency in the same plane, and the rolling problem becomes that of 


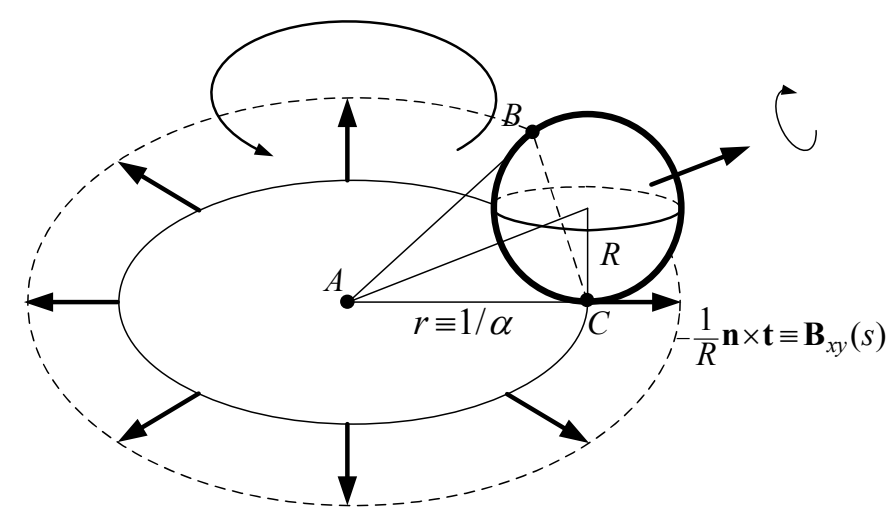

FIG. 1. The lollipop, or a sphere rolling counterclockwise on a circle of radius $r$ corresponds to a spin $1 / 2$ precessing on a magnetic field that rotates in the $x y$ plane.

a sphere of radius $R=1 / B$ rolling counterclockwise on a circle of radius $r=1 / \alpha$ (see Figure 4). In this setting we only have two controls.

In turn, this corresponds to a time (or arc length) dependent Hamiltonian $H=$ $-\mathbf{B} \cdot \mathbf{S}$, which can be solved by noting that

$$
2 \mathbf{B} \cdot \mathbf{S}=\left(\begin{array}{cc}
0 & B e^{-i \alpha s} \\
B e^{i \alpha s} & 0
\end{array}\right)=U^{*}\left(\begin{array}{cc}
0 & B \\
B & 0
\end{array}\right) U,
$$

with

$$
U=\left(\begin{array}{cc}
e^{i \alpha s / 2} & 0 \\
0 & e^{-i \alpha s / 2}
\end{array}\right)
$$

Substituting the above relations in (6) we obtain a time independent equation for the coefficients $\tilde{\chi}(s)=(\tilde{a}, \tilde{b})=\left(e^{i \alpha s / 2} a, e^{-i \alpha s / 2} b\right)$

$$
i \frac{d}{d s}\left(\begin{array}{c}
\tilde{a} \\
\tilde{b}
\end{array}\right)=-\frac{1}{2}\left(\begin{array}{cc}
\alpha & B \\
B & -\alpha
\end{array}\right)\left(\begin{array}{c}
\tilde{a} \\
\tilde{b}
\end{array}\right) \equiv \tilde{H}\left(\begin{array}{c}
\tilde{a} \\
\tilde{b}
\end{array}\right) .
$$

Transformations (16) and (17) correspond to transforming to a frame that rotates with angular velocity $\alpha$ [25]. When transforming to the rotating frame, the angular velocity acquires a component $\alpha=1 / r$ in the $z$ direction and the frequency of rotation in the rotating frame is

$$
\Omega=\sqrt{B^{2}+\alpha^{2}}=\frac{1}{r R} \sqrt{r^{2}+R^{2}}
$$


This can be seen in the spinor language by noting that, since $\tilde{H}$ in Eq. (18) is time-independent, the solutions are

$$
\begin{aligned}
\tilde{\chi}(s) & =e^{\frac{i}{2} s\left(\begin{array}{cc}
\alpha & B \\
B & -\alpha
\end{array}\right)} \tilde{\chi}(0) \\
= & {[\cos (\Omega s / 2)+i \vec{\sigma} \cdot \mathbf{m} \sin (\Omega s / 2)] \tilde{\chi}(0), }
\end{aligned}
$$

with $\pm \Omega=\sqrt{B^{2}+\alpha^{2}}$ (twice) the eigenvalues of $\tilde{H}$ and $\mathbf{m}=(r, 0, R) / \sqrt{r^{2}+R^{2}}$ a unit vector with its $x$ axis in the direction AC. Notice the presence of the factor of 2 in the relation between the eigenvalues of $H$ and the corresponding rotational frequencies of the rolling problem. This comes from the factor $1 / 2$ that emerges naturally in the mapping to the spin problem of Eq. (6). It is important to keep track of this factor in switching to each side of the isomorphism. Equation (20) describes a rotation at a rate $\Omega$ with respect to an axis in the direction of the "stick" of a lollipop (the direction joining $A$ to the center of the sphere (see Fig. (4)). Notice that solving for the evolution by exponentiating $\tilde{H}$ is possible because $\tilde{H}$ does not depend on $s$. If there is an $s$-dependence and the matrices $\tilde{H}$ at different $s$ do not commute the solution is a "time ordered" exponential that in general is not exactly solvable.

After the lollipop completes a circle, the angle $\delta$ of rotation is

$$
\delta=\frac{2 \pi}{\alpha} \Omega=2 \pi \sqrt{1+\left(\frac{r}{R}\right)^{2}}
$$

Notice that, when $R \ll r$ the angle of rotation is $\delta \approx 2 \pi r / R$, corresponding to rolling in a line of length equal to the perimeter of the circle.

We see that, after traveling on a circle the sphere is rotated by $2 \pi \Omega / \alpha$ with respect to an axis tilted with respect to the plane; this is the nonholonomy treated in [10] and [24].

The angle of rotation $\delta$ (of both the spin and the lollipop) has a simple geometric interpretation: when the lollipop rolls, the point of contact $C$ moves on the circular rim of the cone $A B C$ (see Figure 4). At the same time, the point $C$ "paints" on the sphere a circle of diameter $B C=2 r R / \sqrt{r^{2}+R^{2}}$. (This is easily calculated with simple geometrical considerations from Figure 4.) This means that after a revolution of length $2 \pi r$ the angle rotated is $2 \pi r /(B C / 2)$ from which Eq. (21) follows immediately.

At this point we consider Brockett's question mentioned in the introduction. Notice first that, as the sphere rolls on a circle, the velocity at the top of the sphere is twice the velocity $\mathbf{V}$ at the center of the sphere. Since each point of the plane on top of the sphere describes a circle of radius $R_{1}$, the velocity $\mathbf{V}_{P}$ of the plane also describes a circle. Therefore, since the sphere has a rolling condition with the upper plane, then $\mathbf{V}_{P}=2 \mathbf{V}$, meaning that, as the plane describes a circle of radius $R_{1}$ the 
sphere describes a circle of radius $R_{1} / 2$. We can then use the above analysis to relate the rotatoin of the plate to the rotation of the ball.

Notice also that for $s=2 \pi / \alpha$ the spinor $\chi$ changes sign due to the $1 / 2$ factor in the transformation. Nevertheless, since the mapping of (7) is quadratic in $a$ and $b$, changing their signs corresponds to the same values $(x, y, z)$ for the orientations. More specifically, the quantities $a$ and $b$ determine univocally $x, y$ and $z$, but the reverse is not valid: the quantum evolution determines univocally the classical evolution but there is some ambiguity in going from the classical to the quantum case. For example if we perform the "gauge transformation" $(a, b) \rightarrow e^{i \phi(s)}(a, b)$ the mapping to the $\mathbf{X}$ coordinate remains unchanged.

Note also that we have

TheOREm 2. Rolling of the sphere on a circle on any plane corresponds to geodesic motion in $s$ in a coordinate system rotating with the sphere.

5. Rolling on a Curved Surface. In this section we extend the treatment of rolling on a plane to rolling on a curved surface (See Figure 5). If we call $\mathbf{X}_{P}$ the coordinate of the contact point, the coordinate $\mathbf{X}_{c}$ of the center of the sphere is:

$$
\mathbf{X}_{c}=\mathbf{X}_{P}+R \mathbf{n}
$$

and its velocity is given by

$$
\begin{aligned}
\dot{\mathbf{X}}_{c} & =\dot{\mathbf{X}}_{P}+R \dot{\mathbf{n}}, \\
& =\left(\mathbf{t}+R \frac{d \mathbf{n}}{d s}\right) \frac{d s}{d t} .
\end{aligned}
$$

The rolling condition is that the velocity of a point of the sphere in contact with the surface is zero (See Eq.(1)):

$$
\vec{\omega} \times(\mathbf{n} R)=\dot{\mathbf{X}}_{c}
$$

Again, taking the cross product with $\mathbf{n}$ on both sides of the equation above we obtain

$$
\vec{\omega}=\frac{1}{R} \mathbf{n} \times \dot{\mathbf{X}}_{c}
$$

We now replace (23) in (25), and use the fact that, for a curved surface, the variation of the normal is given by

$$
\frac{d \mathbf{n}}{d s}=-\kappa_{n} \mathbf{t}-\tau_{r} \mathbf{u}
$$

with $\kappa_{n}$ the normal curvature and $\tau_{r}$ the torsion of the curve, both evaluated at the contact point. We obtain 


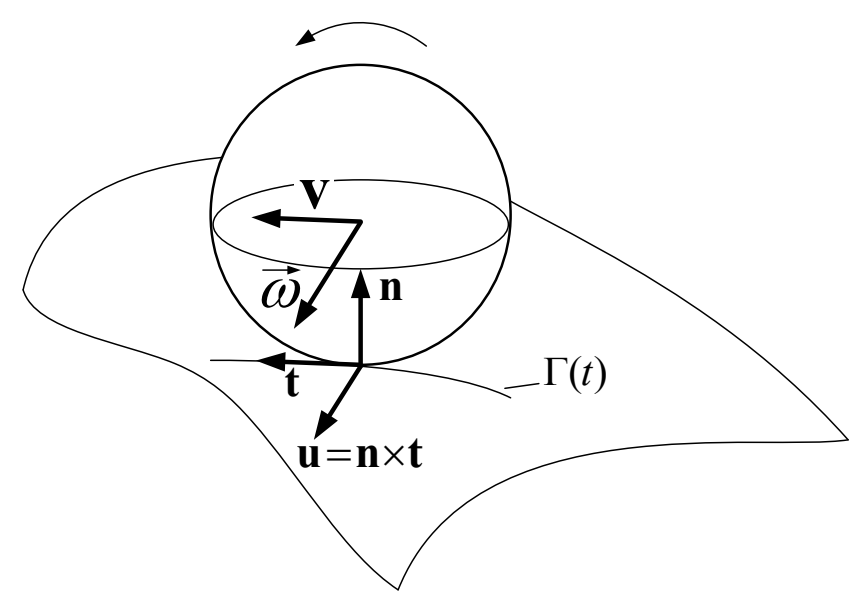

FIG. 2. Sphere rolling along a curve $\Gamma$ of zero torsion (meaning that the velocity of the center of the sphere is parallel to the tangent of the curve at the contact point).

$$
\vec{\omega}=\left[\frac{1}{R}\left(1-\kappa_{n} R\right) \mathbf{u}+\tau_{r} \mathbf{t}\right] \frac{d s}{d t} .
$$

The discussion for the planar case extends to the curved surface, and the rolling of the sphere is equivalent to a spin $1 / 2$ precessing on a magnetic field $\mathbf{B}(s)$ given by

$$
\mathbf{B}(s)=-\left[\frac{1}{R}\left(1-\kappa_{n} R\right) \mathbf{u}+\tau_{r} \mathbf{t}\right],
$$

with the arc length $s$ playing the role of time. This is turn gives us the control vector fields for rolling on the surface. In the following section, as an example of this formulation we consider rolling on a spherical surface.

6. Sphere Rolling on a Spherical Surface. In this section we consider a sphere of radius $R$ rolling on a second sphere of radius $r$. The rolling line will be a parallel of latitude $\pi / 2-\theta$ (see Figure 3 ). This means that the normal curvature is constant $1 / r$, and also that the torsion is zero. The magnetic field for the corresponding spin problem is therefore:

$$
\mathbf{B}_{ \pm}(s)=-( \pm)\left[\frac{1}{R}\left(1 \pm \frac{R}{r}\right) \mathbf{u}\right]=-( \pm) \frac{1}{\widetilde{R}_{ \pm}} \mathbf{u}
$$




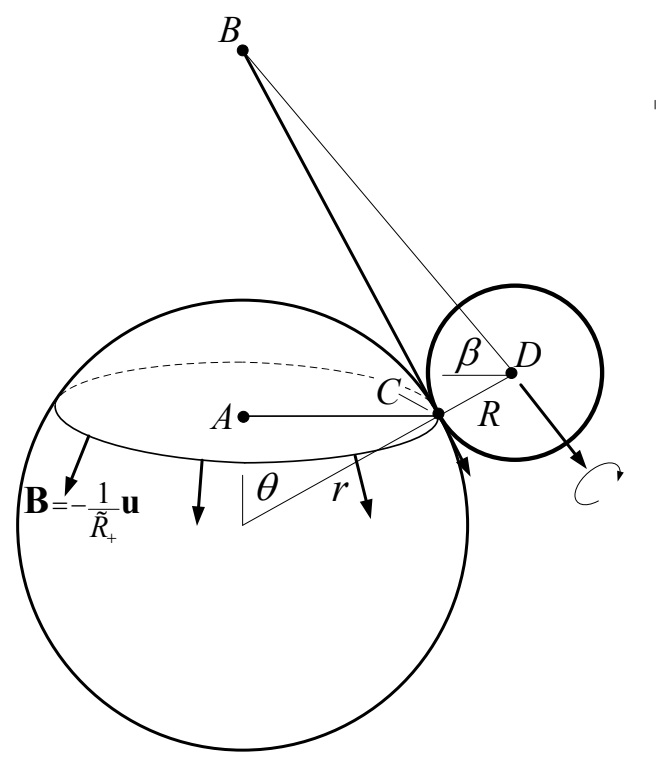

FIG. 3. Sphere rolling on a sphere.

with $\widetilde{R}_{ \pm}=r R /(r \pm R)$ a reduced radius and the plus and minus signs refer to the rolling outside and inside of the sphere of radius $r$ respectively.

For a sphere rolling on a parallel, the instantaneous angular velocity (and the magnetic field) describes a cone forming an angle $\theta$ with the vertical. The total arc length of the parallel is $r \sin \theta$ meaning that the vector $\mathbf{u}$ rotates with angular frequency $\alpha$ given by $\alpha=1 /(r \sin \theta)$. The corresponding magnetic field is therefore

$$
\begin{aligned}
\mathbf{B}_{ \pm}(s) & =\left(B_{x}, B_{y}, B_{z}\right)_{ \pm} \\
& =( \pm) \frac{1}{\widetilde{R}_{ \pm}}(\cos \theta \cos \alpha s, \cos \theta \sin \alpha s,-\sin \theta)
\end{aligned}
$$

with the term $\mathbf{B} \cdot \mathbf{S}$ in the corresponding Hamiltonian given in this case by

$$
\mathbf{B} \cdot \mathbf{S}= \pm \frac{1}{2} \frac{1}{\widetilde{R}_{ \pm}}\left(\begin{array}{cc}
-\sin \theta & \cos \theta e^{-i \alpha s} \\
\cos \theta e^{i \alpha s} & \sin \theta
\end{array}\right) .
$$

This again is an exactly solvable Hamiltonian that was first studied by Rabi

Using the same transformation matrix of Eq. (17) the above Hamiltonian can be rendered time independent. We write it in the following form

$$
\tilde{H}=-\frac{1}{2}\left(\begin{array}{cc}
-B_{ \pm} \sin \theta+\alpha & B_{ \pm} \cos \theta \\
B_{ \pm} \cos \theta & B_{ \pm} \sin \theta-\alpha
\end{array}\right)
$$


with $B_{ \pm}=1 / \widetilde{R}_{ \pm}$.

The eigenvalues of $\tilde{H}$ are $E_{ \pm}=\Omega_{ \pm} / 2$ with

$$
\Omega_{ \pm}=( \pm) \frac{1}{\widetilde{R}_{ \pm}} \sqrt{1-\frac{2 \widetilde{R}_{ \pm}}{r}+\left(\frac{\widetilde{R}_{ \pm}}{r \sin \theta}\right)^{2}}
$$

with the spinor precessing, in the rotating frame, around an axis that forms an angle $\beta$ (see Figure 3 ) with the $x y$ plane, with

$$
\tan \beta=\tan \theta-\frac{R}{r+R} \frac{1}{\sin \theta \cos \theta}
$$

The second term in (34) reflects the fact that the small sphere rotates instantaneously on the tangent plane that contains $B C$ (see Figure 3). Equation (34) can be easily derived by simple geometric considerations from Figure (3).

After a complete revolution the angle or rotation $\delta$ is

$$
\delta_{ \pm}=2 \pi r \sin \theta \Omega_{ \pm}
$$

After a little algebra we obtain

$$
\delta_{ \pm}= \pm 2 \pi \cos \theta \sqrt{1+\left(\frac{r \tan \theta}{R}\right)^{2}}
$$

Notice that, if we compare with the rotation in a plane from Eq. (19), the rotation corresponds to rolling on a circle of radius equal to that of the unfolded cone tangent to the parallel (See Fig. 3). The angle of rotation along that circle is not $2 \pi$ but $2 \pi \cos \theta$. This geometric factor is the same that appears in Foucault's pendulum and in Berry's phase for a spin precessing on a cone (we will come back to this point below). Also, notice that when $r=R$ the angle of rotation is always $2 \pi$ independent of latitude.

We finish this section with a discussion of the differences and similarities between the Berry phase for a precessing spin $1 / 2$ in the adiabatic approximation and the rolling of two spheres.

The Hamiltonian for a spin in a magnetic field that precesses along the $z$ axis at frequency $\alpha$ is given by (32), where in principle $\alpha$ and $B_{0}$ are independent parameters. If $\alpha \ll B_{0}$ (the adiabatic approximation) the eigenvalues (eigenfrequencies) of $\widetilde{H}$ are

$$
\Omega \simeq \sqrt{B_{ \pm}^{2}-2 \alpha B_{ \pm} \sin \theta} \simeq B_{ \pm}-( \pm) \alpha \sin \theta .
$$

After a period of time $2 \pi / \alpha$ the change $\Delta \phi$ in the phase of the spin is

$$
\Delta \phi=2 \pi \frac{B_{ \pm}}{\alpha}-( \pm) 2 \pi \sin \theta .
$$


The first term is the dynamical phase and the second is a purely geometrical one, independent of the parameters $B_{0}$ and $\alpha$, and given by (half) the solid angle described by the field.

For the rolling sphere we can also study an "adiabatic approximation" since $\alpha \ll$ $B_{0}$ corresponds to $r \gg R$. In other words, in general the adiabatic approximation will correspond to the radius of the rolling sphere much smaller than the radius of curvature of the surface. On the other hand, in contrast with the spin case, the frequency of rotation $\alpha=1 / r \sin \theta$ "knows" about the latitude and the curvature. So we expect some differences and some similarities. Replacing the values of $B_{ \pm}=$ $\pm 1 / \widetilde{R}_{ \pm} \equiv( \pm) 1 / R \pm 1 / r$ in (38) we obtain the angle of rotation of the sphere in each case (in the adiabatic approximation)

$$
\begin{aligned}
\Delta \phi_{ \pm} & = \pm 2 \pi r \sin \theta\left(\frac{1}{R} \pm \frac{1}{r}\right)-( \pm) 2 \pi \sin \theta . \\
& = \pm 2 \pi \frac{r \sin \theta}{R}
\end{aligned}
$$

Notice that there is a cancelation of the geometric phase for rolling. In the spin problem, the frequency $\omega$ of rotation of the field ( $\alpha$ for rolling) and the magnitude of the field $B_{ \pm}$are independent and therefore the total angle of rotation is given by Eq.(38), with the second term a purely geometric term independent of the parameters of the problem. In the rolling case the frequency and the field are not independent, and the "dynamical" phase contains a term that cancels the geometric one. As a result, the total rotation is given by a magnitude that depends on the parameters of the problem, which, in the spin language corresponds to the dynamical phase only. This cancelation is a general result that we will visit in the next section.

Again, from above we have

THEOREM 3. Rotation of a sphere on a line of lattitude of a spherical surface corresponds to geodesic motion in $s$ in the rotating frame.

In the next section we discuss the general connection between rolling and the Berry phase for spins in the adiabatic approximation.

7. The Adiabatic Approximation and Rolling on a Curved Surface. In this section we compare the equivalence between the adiabatic approximation for a spin precessing in a magnetic field that changes direction at a slow rate and rolling on a surface. In the spin case, the dimensionless parameter controlling the approximation is the ratio of the instantaneous frequency (proportional to the instantaneous magnitude of the field) with the rate at which it's direction is changing.

In the rolling case the instantaneous frequency corresponds to the magnitude of $\mathbf{B}(s)$ and the rate of change in its direction is related to the normal curvature and to the curve's torsion.

In the adiabatic approximation for spins [27], one works in an "instantaneous" 
basis, treating first $s$ (time) as a parameter and solving the eigenvalue equation as though the problem were static:

$$
H(s) \chi(s)=E(s) \chi(s) \equiv \frac{\Omega(s)}{2} \chi(s) .
$$

Then the general solution is written as linear combinations of the instantaneous eigenstates. As a result, in the adiabatic approximation, the spinor at time $s$ is given by

$$
\chi(s)=e^{i \gamma(s)} e^{i \int_{0}^{s} d s^{\prime} E\left(s^{\prime}\right)} \chi(0) .
$$

The argument of the second exponential above represents the dynamic phase, which involves the integral of (half) the following angular frequency:

$$
\begin{aligned}
\Omega(s) & =|\mathbf{B}(s)|=\frac{1}{R} \sqrt{\left[1-\kappa_{n}(s) R\right]^{2}+[\tau(s) R]^{2}} \\
& \simeq \frac{1}{R}-\kappa_{n}(s)
\end{aligned}
$$

This can be seen, for example from Equation (31): the eigenvalues of $\mathbf{B} \cdot \mathbf{S}$ with $s$ treated as a parameter are $\pm|\mathbf{B}(s)| / 2$.

The (instantaneous) direction of the field is in the direction $\mathbf{u}_{B}$ given by

$$
\mathbf{u}_{B}=\frac{\mathbf{B}(s)}{|\mathbf{B}(s)|}=-\frac{\left(1-\kappa_{n} R\right) \mathbf{u}+\tau_{r} \mathbf{t}}{\sqrt{\left(1-\kappa_{n} R\right)^{2}+\tau_{r}^{2}}}
$$

In general, the eigenvalues of a Pauli matrix in an arbitrary direction $\mathbf{u}_{B} \cdot \vec{\sigma}$ given by the unit vector $\mathbf{u}_{B}=\left(u_{x}, u_{y}, u_{z}\right)$ are \pm 1 . This is verified by noting that (defining $\left.u_{x}+i u_{y}=\rho e^{i \phi}\right)$

$$
\left(\mathbf{u}_{B} \cdot \vec{\sigma}\right) \chi_{ \pm}\left(\mathbf{u}_{B}\right)=\left(\begin{array}{cc}
u_{z} & \rho e^{-i \phi} \\
\rho e^{i \phi} & -u_{z}
\end{array}\right) \chi_{ \pm}\left(\mathbf{u}_{B}\right)= \pm \chi_{ \pm}\left(\mathbf{u}_{B}\right)
$$

with $\chi_{ \pm}\left(\mathbf{u}_{B}\right)=\left(1, \pm\left(1-u_{z}\right) e^{ \pm i \phi} / \rho\right)$. Notice that the dependence of $\chi$ on $s$ is through the orientation of $\mathbf{u}$.

The first term of (41), the geometric phase $\gamma$, is the Berry phase, and is given by $[28]$

$$
\dot{\gamma}(s)=i \chi\left(\mathbf{u}_{B}(s)\right)^{\dagger} \frac{d}{d s} \chi\left(\mathbf{u}_{B}(s)\right) .
$$

Without loss of generality we express $\mathbf{u}_{B}$ in polar coordinates $\mathbf{u}_{B}=(\cos \theta \cos \phi$, $\cos \theta \cos \phi, \sin \theta$ ), where the quantization axis $z$ is perpendicular to the instantaneous 
plane of motion of the center of mass of the rolling sphere. This means that the normalized spinor is:

$$
\chi\left(\mathbf{u}_{B}(s)\right)=\left(\begin{array}{c}
\frac{\cos \theta(s)}{\sqrt{1+\sin \theta(s)}} \\
\sqrt{1+\sin \theta(s)} e^{-i \phi(s)}
\end{array}\right) .
$$

From the above expression and (45) we can compute the geometric phase:

$$
\dot{\chi}=i \chi^{\dagger} \frac{d}{d s} \chi=\frac{1+\sin \theta}{2} \frac{d \phi}{d s} .
$$

Here $d \phi$ is the angle of rotation of the center of mass of the sphere with respect to an instantaneous axis of rotation. The first term of the right hand side is $2 \pi$ after integration on a closed circuit. And the second term cancels the curvature term from Eq. (42). This results from the identity

$$
\frac{d \phi}{d s}=\frac{\kappa_{n}}{\sin \theta} .
$$

Our final result is that, as anticipated in the two spheres case, in general there is no Berry phase for rolling as a result of the above cancelation:

$$
\begin{aligned}
\delta_{ \pm} & = \pm\left(\int_{0}^{s} d s^{\prime} \Omega\left(s^{\prime}\right)+2 \gamma(s)\right) \\
& = \pm \frac{L}{R}+2 \pi
\end{aligned}
$$

Note that, if we specify this result to the sphere rolling on the parallel of a sphere of radius $r$, we have $L=2 \pi r \sin \theta$. Replacing these in Eq. (42) we obtain the result of Eq. (39) as expected. The discrepancy of the (unimportant) factor $2 \pi$ results from the fact that the treatment in the present section is in the rest frame and that of section 6 is in the rotating frame. The plus and minus signs corresponds both to the two senses of traveling the circuit and the two sides of the surface on which the sphere can roll.

8. Rolling on a Cornu Spiral and the Landau-Zener Problem. In this section we consider a "magnetic field" of constant magnitude $B_{0}$ varying on the $x y$ plane as

$$
\mathbf{B}=B_{0}(\cos \phi(s), \sin \phi(s), 0)=-\vec{\omega},
$$

which corresponds to the angular frequency rotating with the (varying) frequency $\phi(s)$ in the plane. The rolling problem becomes that of a sphere of radius $R=1 / B_{0}$ rolling on a planar curve of local curvature given by

$$
\kappa(s)=\dot{\phi} \equiv \frac{d \phi}{d s}
$$


This corresponds to a time (or arc length) dependent Hamiltonian $H=-\mathbf{B} \cdot \mathbf{S}$, which can be solved by noting that

$$
\begin{aligned}
\mathbf{B} \cdot \mathbf{S} & =\left(\begin{array}{cc}
0 & B_{0} e^{-i \phi(s)} \\
B_{0} e^{i \phi(s)} & 0
\end{array}\right) \\
& =U^{*}\left(\begin{array}{cc}
0 & B_{0} \\
B_{0} & 0
\end{array}\right) U
\end{aligned}
$$

with

$$
U=\left(\begin{array}{cc}
e^{i \phi(s) / 2} & 0 \\
0 & e^{-i \phi(s) / 2}
\end{array}\right)
$$

Substituting the above relations in (6), and using (51) we obtain a time dependent equation for the coefficients $\tilde{\chi}(s)=(\tilde{a}, \tilde{b})=\left(e^{i \phi / 2} a, e^{-i \phi / 2} b\right)$

$$
i \frac{d}{d s}\left(\begin{array}{c}
\tilde{a} \\
\tilde{b}
\end{array}\right)=-\frac{1}{2}\left(\begin{array}{cc}
\kappa(s) & B_{0} \\
B_{0} & -\kappa(s)
\end{array}\right)\left(\begin{array}{c}
\tilde{a} \\
\tilde{b}
\end{array}\right) \equiv \tilde{H}\left(\begin{array}{c}
\tilde{a} \\
\tilde{b}
\end{array}\right) .
$$

We have obtained the nice result that rolling on a planar curve is isomorphic to spin precession on a magnetic field that is constant in (some direction of) the $x y$ plane, and with a $z$ component that varies in time according to the local curvature. For example, this means that rolling on a Cornu spiral (a curve whose curvature is proportional to the arc length), defined as

$$
\phi(s)=a s^{2} / 2,
$$

with $a$ a constant, corresponds to the Landau-Zener[14] problem of a spin in a magnetic field whose $z$ component varies linearly with time $B_{z}=a s$ and a constant $x y$ coupling of magnitude $1 / R$. The prototypical question in the Landau-Zener problem is the flipping of a spin that starts, for $t \rightarrow-\infty$, in a well defined orientation in the $z$ direction. In the language of section 2 this means that $|a(-\infty)|=1$, and $|b(-\infty)|=0$. In the sphere isomorphism, the level splitting $\Delta E$ (See Figure 4) increases in time (arc length $s$ ) as $\Delta E=a s$ and the level coupling is $B_{0}=1 / R$, constant in time. The problem is also called the avoided level crossing. The name comes from the fact that, when $B_{0}=0$ the levels for spin up and down cross at $s=0$. The remarkable result obtained by Zener is that the probability of the spin remaining up after the evolution is, in our notation

$$
|a(\infty)|=e^{-\pi / 2 a R^{2}} .
$$

In [5] we show show that the non-adiabatic limit when the levels are crossed very fast (which for rolling corresponds to a sphere much larger than the size of the Cornu spiral) can be obtained in a simple way using the rolling picture. 

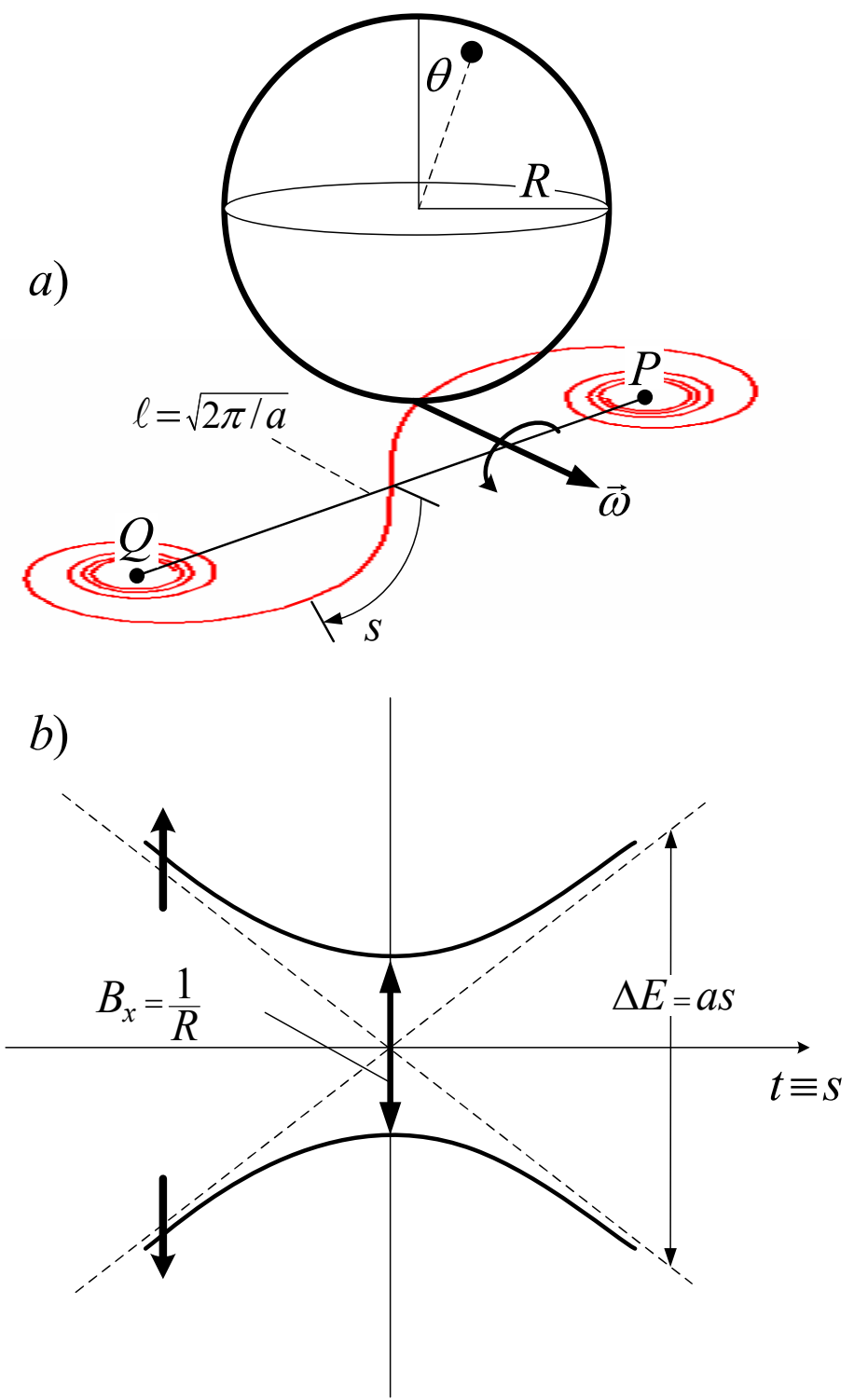

Fig. 4. Equivalence between a) rolling on a Cornu spiral and b) the Landau-Zener problem of the spin flip probability on a time dependent field.

Note that the control problem in this case does not correspond to optimal motion in the sense desribed earlier.

9. Acknowledgments. We thank Michael V. Berry for useful comments on the manuscript and for pointing us to Ref [12]. We also thank Paul Berman, Gil Bor and Richard Montgomery for helpful remarks. A.G.R thanks the Research Corporation, and A.M.B. thanks the National Science Foundation for support. 


\section{REFERENCES}

[1] J. Baillieul, Some Optimization Problems in Geometric Control Theory, Ph.D. thesis, Harvard University (1975).

[2] J. Baillieul, Geometric methods for nonlinear optimal control problems, J. of Optimization Theory and Applications, 25(1978), pp. 519-548.

[3] A. M. Bloch, P. S. Krishnaprasad, J. E. Marsden, and G. Sánchez De Alvarez, Stabilization of rigid body dynamics by internal and external torques, Automatica 28(1992), $745-756$.

[4] A. M. Bloch, R. Brockett, And P. Crouch, Double bracket equations and geodesic flows on symmetric spaces, Comm. Math. Phys. 187(1997), 357-373.

[5] A. Rojo And A. M. BLOCH, The rolling sphere and the quantum spin and a simple view of the Landau-Zener problem (2010), to appear in the American Journal of Physics.

[6] W. Wong and J. Baillieul, Cost and Complexity of Distributed Choices, preprint (2009).

[7] R. W. Brockett, Nonlinear control theory and differential geometry, in: Proc. International Congress of Mathematicians, 1357-1368 (1983).

[8] R. Montgomery, How much does a rigid body rotate? A Berry's phase from the 18th century, Am. J. Phys, 59(1991), pp. 394-398.

[9] M. Levy, Geometric Phases in the Motion of Rigid Bodies, Arc. Rational. Mech. Anal. 122(1993), pp. 213-229.

[10] B. D. Johnson, The nonholonomy of the rolling sphere, The American Mathematical Monthly, 114(2007), pp. 500-508.

[11] R. Brockett And L. Dai, Non-holonomic kinematics and the role of elliptic functions in constructive controllability, in Z. Li and J. Canny (eds), Nonholonomic Motion Planning, Kluwer, 1993.

[12] M. V. Berry And J. M. Robins, Classical Geometrical forces of reaction: an exactly solvable model. Proc. Roy. Soc. Lond. A 442(1993) pp. 641-658.

[13] J. H. Hannay, Angle Variable Holonomy in Adiabatic Excursions of an Integrable Hamiltonian, J. Phys. A18(1985) pp. 221-230. Also reprinted in [23].

[14] C. Zener, Non-adiabatic Crossing of Energy Levels. Proc. Roy. Soc. Lond. A 137(1932), pp.692702.

[15] H. Guggenheimer, Differential Geometry (Chapter 10), Surfaces, Dover (1977).

[16] L. D. Landau and E. M. Lifshitz, Mechanics, Third Edition, p. 123, Butterworth-Heineman, Amsterdam, (2003).

[17] A. M. Bloch, ,with J. Baillieul, P. Crouch, and J.E. Marsden, Nonholonomic Mechnaics and Control, Springer Verlag, 2003.

[18] G. B. Arfken and H. J. Weber, Mathematical Methods for Physicists, Academic Press, pp. 232-236 (1995).

[19] R. P. Feynman, F. L. Vernon, and R. W. Hellwarth, Geometrical Representation of the Shrödinger Equation for Solving Maser Problems, J. Appl. Phys, 28(1957), pp. 49-52.

[20] F. Bloch, Nuclear Induction, Phys. Rev. 70( 1946), pp. 460-474.

[21] I. I. RABI, Space Quantization in a Gyrating Magnetic Field, Phys. Rev. 51(1937), pp. 652-654.

[22] See for example F. Ansbacher, A note on the equivalence of the classical motion of rigid bodies with prescribed angular velocities and the quantum mechanical solutions for paramagnetic atoms in external fields, J. Phys. B: Atom. Molec. Phys. 6(1973), pp. 1616-1619; H. Urbantke, Two-level systems: States, phases and holonomy, Am. J. Phys. 59(1991), pp. 504-509, and D. J. Siminovitch, Rotations in NMR: Part I. Euler Rodrigues Parameters and Quaternions, Concepts Magn. Reson., 9(1997), pp. 149-171.

[23] Geometric Phases in Physics, edited by A. Shapere and F. Wilczek (World Scientific, Singapore, 1989). 
[24] T. Iwai And E. Watanabe, The Berry phase in the plate-ball problem, Phys. Lett. A, 225(1997) pp. 183-187.

[25] See for example C. P. Slichter, Principles of Magnetic Resonance, Third Edition, pp. 25-35 (Springer-Verlag, Berlin, 1990)

[26] For more ellaborate treatments see G. Bor and R. Montgomery, $G_{2}$ and the Rolling Distribution, preprtint arXiv:math/0612469v1, and J.E. Marsden, R. Montgomery, and T.S. Ratiu, Reduction, Symmetry and Phase in Mechanics, Memoirs of the American Mathematical Society, Providence, RI, vol 436, (1990).

[27] J. J. SakuraI, Modern Quantum Mechanics, (Addison-Wesley, New York), pp. 464-468

[28] M. V. Berry, Quantal Phase Factors Accompanying Adiabatic Changes. Proceedings of the Royal Society of London, A, 392, pp. 45-56 (1984)

[29] D. J. Montana, The Kinematics of Contact and Grasp, The International Journal of Robotics Research, 7 (1988), pp.17-32.

[30] For a more detailed discussion see B. R. Holstein, The adiabatic theorem and Berry's phase, Am. J. Phys. 57 (1989), pp.1079-1084.

[31] For a related discussion see M. Levi. A 'bicycle wheel' proof of the Gauss-Bonnet theorem. Expositiones Mathematicae, 12(1993), pp.145-164. 